\title{
Implementation about Thread and Internet-based Motion Receiving Imitation Controller for Humanoid
}

\author{
Hee-Jung Byun ${ }^{1}$ and Su Goog Shon ${ }^{1 *}$ \\ ${ }^{1}$ Department of IT Engineering, University of Suwon, IT Building, San 2-2 Wau-ri, \\ Bongdam-eup, Hwaseong-si, South Korea \\ ${ }^{1}$ heejungbyun@suwon.ac.kr, ${ }^{1}$ *sshon@suwon.ac.kr
}

\begin{abstract}
In this paper, the method that allows for the combined control scheme of the imitation and autonomous control for a humanoid robot via Internet is presented. Our approach does not use any prior analytical or mathematical robot modeling (e.g. forward or inverse kinematics). All the way from the human operator with encoders to the humanoid joints, many server motors and encoders are connected with single communication path for the imitation control. The key concept for autonomous control is added to let the robot stabilize by utilization of tracing the CoP (center of pressure) and controlling the ankle of the robot's support foot prior to impact in real time. Thread programming technology is introduced to enhance the control and operation speed up, and explained how to operate joints. The experimental results demonstrate that the humanoid robot can successfully imitate the motions of the operator and can walk stably by both utilizing the CoP and controlling the robot's ankles.
\end{abstract}

Keywords: control communication, thread, humanoid, imitation control, Internet

\section{Introduction}

In general, robots do simple and dangerous jobs in place of people. For instance, they are engaged in simple assembly processes in manufacturing plants, exploration of the deep sea, and dismantling explosives. It has been expected that the number of application areas of robots could be increased with the remote control of robots. Especially, humanoid robot based on remote control has wide range of applications such as entertainment and surveillance. Flexible mobility and intelligence are very much required for such robotics applications.

Even though many studies all around the world have been done to let biped humanoids have flexible mobility and intelligence, intelligence of humanoids are still very too low to do smart jobs. It will not be possible soon for humanoid to get smart enough to think or decide by himself/herself.

Necessities to control biped humanoids by human are increasing. Then, a human controlled biped robot can work for humans in places difficult for human to access such as disaster areas. The human-controlled biped robot would be intelligent enough to do smart jobs because it is controlled by human's intelligence. Even though this type of robot is attractive, there are insufficient research up to this point.

Traditional control method uses remote controllers, named RC, based on RF technology. Since N. Tesla demonstrated the first radio-controlled boat in 1898 and R. Goertz demonstrated a bilateral manipulator in 1954 [1], remotely operated robots have been widely designed. Teleoperation for a robot is controlled from a secure distance by a human operator, and at the same time, the robot should avoid

${ }^{*}$ Corresponding Author 
being harmed by incorrect user commands (self-protection) and avoid causing damage (avoid collisions). Finally the teleoperation should provide some feedback to the operator and automatically identify incidents, sending back information to the user for stable control.

In order to enhance the mobility of robots, wheeled robots have been considered. Wheeled robots, even though they possess superior mobility, have difficulty moving over debris. In addition, wheeled robots cannot decide by themselves on how to get through those kinds of obstacles because of their low intelligence. So, teleoperation technology has been adopted to compensate for intelligence. Research concerning the teleoperation of robots have usually been done in wheeled robots such as Pioneer 3AT, Erratic EraMobi, and Surveyor SRV-1 Blackfin [2]. Since Honda began to develop humanoid robots in 1986, there have been attempts to teleoperate bipedal robots with remote controllers (RC). However, it turned out that they are low in DOF and possessed limited motion control. One disadvantage using RC is low degree of freedom with 8 DOFs at most and another is a short distance control range. Various types of robots able to meet consumer needs have been designed with the ability to be controlled via wired/wireless communication. In order to teleoperate a humanoid, several technologies such as console, joy stick, special interface, motion capture suit, etc., have been utilized.

With a teleoperation console, an operator inputs the robot's path and actions such as power on, stand by, simple tasks, and power off. The robot is then able to move on a planned path autonomously and to perform simple operations via wireless teleoperation, as system developed in 1998 [3,4]. A joystick master device was also developed in 2002 for the teleoperation of whole body motions of humanoid robots [5]. Using a joy stick with the assumption that the operator understands environments, the operator can then move a humanoid robot with only a single three-axis joystick with a button via teleoperation, which provides directional commands to be interpreted. Here, the robot computes trajectories that satisfy kinematic and dynamic constraints to safely execute the desired motion [6]. Robots in LRM Lab. have been teleoperated by humans with remote controllers and a communication network since 2009 [7]. Keio University has shown that bipedal robots can be controlled by recognizing touch inputs of gestures similar to those used in touch-screen smartphones [8]. A. Senior and S. Tosunnoglu have presented how to control bipedal robots by transmitting commands from a computer with a serial communication connection to a bipedal robot [9]. Additionally, Bluetooth can be used to control a robot remotely. In terms of real-time operating system for robot, European automotive operating standard (OSEK/VDX) has also been introduced in order to increase the reliability and stability of the robot [10].

The imitation control is basically to copy human body motions. Motion capture has had a significant impact on robotics, being used for not only teleoperation but also for improving humanoid robots. In order to trace human body motions, several sensors such as gyroscope, acceleration sensors [11,12], magnetic marking systems [13], small inertial sensors [14], force sensors [15], or optical tracking [16] are utilized to specific areas of the body. Early approaches used inertial measurement units [14, 19], photo detectors [16,20], and shape tape [21]. In recent years, many new motion capture products have come onto the market, ranging from the cheap but somewhat limited (e.g. Microsoft Kinect, Nintendo Wii) to the (relatively) expensive but highly accurate Xsens MVN full-body motion capture suit [22]. In one instance, a master interface worn by a human operator that served to map the lower limb joint angles of the human operator to those of the humanoid robot was designed and obtained rough terrain adaptability by means of teleoperation in 2004 [23]. T. Liu et al. especially have presented that a robot's gait can be estimated after applying a fuzzy inference engine to measurement data of angular speed and 
acceleration [11, 12]. Ude et al. has used a magnetic marking systems for the measurement of joint angles [13]. A small inertial sensor system has also been used to measure lower body segment orientations [14]. R. Chalodhorn et al. have said that direct use of motion capture data is more likely to be flexible and intuitive than programming control, though it is kinetically unstable [17]. As another instance, both a real-time 3D human motion capture system using force sensors and a hand tracking device can be used for full-body motion reconstruction [15].

Even though there are different types of sensor systems to trace motion or joint angles for the human operator, they are not a direct method which needs some calculations from indirect measurements, nor a real time method. Direct use of motion capture data is more likely to be flexible and intuitive than programming control, though it is kinetically unstable [18].

In this paper, the method that allows for the combined control scheme with the imitation control and autonomous control for a humanoid robot via Internet is presented. Our approach does not use any prior analytical or mathematical robot modeling (e.g. forward or inverse kinematics). All the way from the human operator with encoders to the humanoid joints, many server motors and encoders are connected with single communication path for the imitation control. The concept for autonomous control is added to let the robot stabilize by utilization of tracing the CoP (center of pressure) and controlling the ankle of the robot's support foot prior to impact in real time. Thread programming technology is introduced to enhance the control and operation speed up, and explained how to operate joints. In the past, experiments are designed and done to show that the combined control scheme using imitation and autonomous control can be used to control a humanoid in real time with the help of the fast thread programming technology.

\section{Design of Internet-Based Imitation and Autonomous Control Scheme}

Proposed control scheme is to use both imitation and autonomous control for teleoperation of robot. The purpose of this research is finally to develop a robot like the avatar that could be moved depending on operator's motion and intent. Imitation control and autonomous control are well known control scheme for a robot so far. Imitation control has advantages that gesture generation for robot control can be accomplished quite fast. However, the imitation control scheme has limitations which can only be applied on flat surface walking. Autonomous robot means that they can perform desired tasks in unstructured environments without continuous human guidance.

The proposed imitation control system consists of several components such as joints motion generator, motion sending controller, motion receiving controller, robot motion controller, and a robot as shown in Figure 1. Here, the joint data from human operator is acquired with the joint motion generator and transferred to the motion receiving controller through the motion sending controller through internet, and the motion receiving controller transfers the operator joint angles to the robot motion controller.

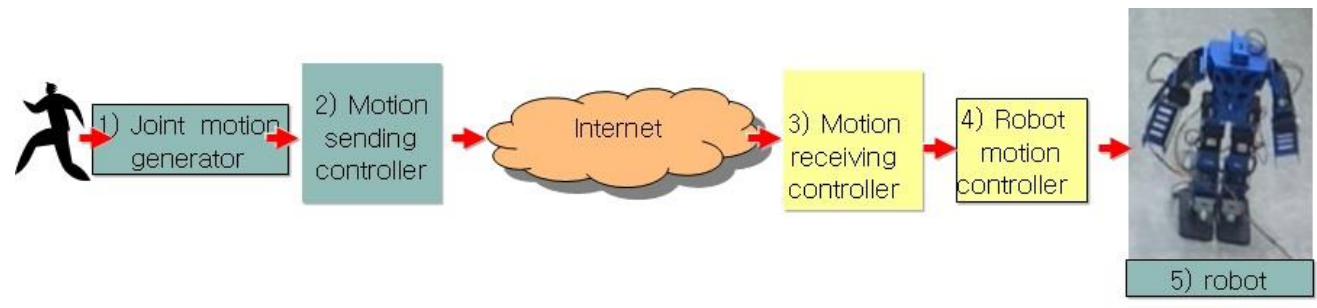

Figure 1. Proposed Robot Control Scheme Using Imitation and Autonomous Control 
The joint motion generator that can capture joint values is built with encoders as shown in Figure 2 and the motion sending controller is made by Windows programming software on a $\mathrm{PC}$ as shown in Figure 3. The motion receiving controller and robot motion controller are built on separate two ARM processors and has imitation and autonomous functions to operate the humanoid robot's joints. All the way from encoders attached to the human operator to the humanoid joints is connected with single communication path. Daisy chained communication path is introduced, which includes all the components in series.

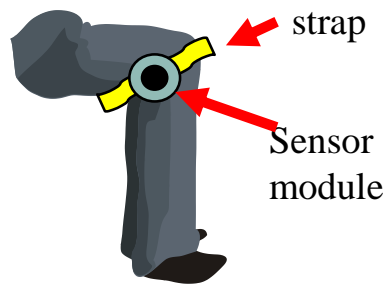

Figure 2. Joint Motion Generator

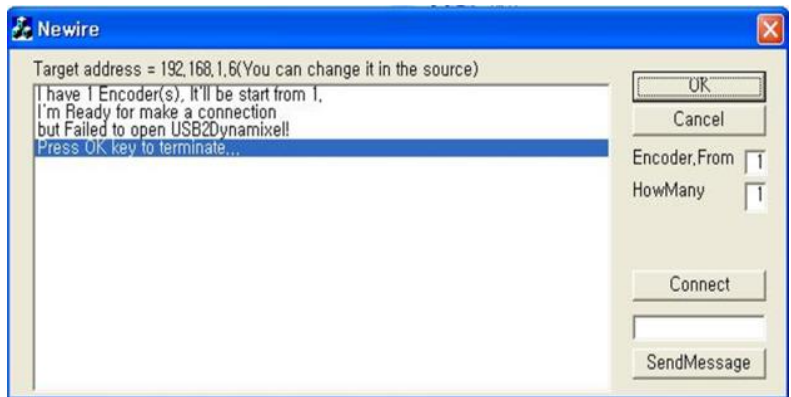

Figure 3. Sample of Motion Sending Controller

Even though there have been some studies concerning imitation control, most of have not yet been applied to actual robot walking. Only virtual robots in cyberspace have yet been able to imitate an operator's motions well. The main problem of the imitation control is that the walking stability of a robot is not guaranteed because the joint angles of the robots are generated by the human operator. Unseen trajectory data can cause instability in robot's walking gait. Imitation control could be used for the more-difficult components of a task, leaving the rest to autonomous control, or as a fallback option if autonomy fails.

\section{Implementation of Thread-Based Motion Receiving Controller}

In this section, a motion receiving controller and a robot controller are discussed. The operator's gait and walking patterns are transmitted to the motion receiving controller via internet. As for the robot motion controller, data for walking trajectory are transmitted to the robot's actuator controllers. As shown in Figure 4, the motion receiving controller do jobs to communicate with the motion sending controller based on client and server. Here the motion sending controller serves as a client and the motion receiving controller as a server. The client and the server communicate to transfer joint angles based on a well-designed communication protocol. The motion sending controller is programmed based on Winsock programming and the motion receiving controller programmed on Linux socket programming. 


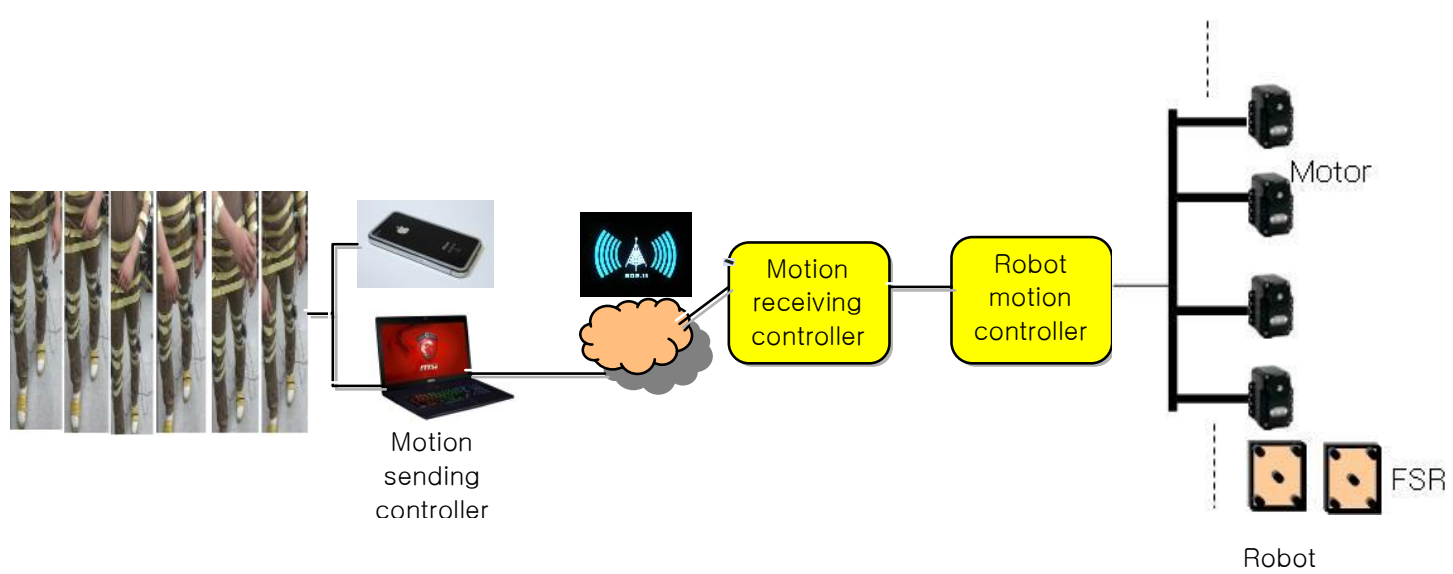

Figure 4. Imitation Control System for a Bipedal Robot

Besides the transfer of motion data, another function of the motion receiving controller is to stabilize the gesture of the robot. The robot motion controller is only to activate each server motor depending on the input command from the motion receiving controller. For this task, the motion receiving controller needs to do some signal processing before sending the motion data. This is a challenging issue to overcome the postural instability caused by dynamics of robots.

From the view of robot kinematics, both forward and inverse kinematic solutions are generally required to control a robot via teleoperation. Traditional approaches to humanoid teleoperation use explicit forward kinematic modeling of both the robot slave and master device to provide mapping between human motion capture data and robot actuator commands. Forward kinematics modeling involves calculating the position and orientation of the end-effector in a three dimensional space given the joint angles (e.g. calculating the position of the hand in XYZ space based upon the values of the shoulder, elbow, and wrist joints). This is easily solved using the geometry of the robot which is specified in the DH parameters.

Conversely, inverse kinematics refers to the use of the kinematics equations of a robot to determine the joint angles given the desired position and orientation of the end-effector. Often, multiple solutions exist for the robot's inverse kinematic calculation if multiple configurations of the robot's joints can result in the same end-effector position. One approach to solving inverse kinematics is through trigonometry, while another is to use Jacobian matrix-based iterative approaches [23]. Both approaches are laborious and changes in robotic hardware require new analysis and kinematic calculations. Another approach to achieve the teleoperation of a humanoid robot such that is able to execute simple tasks is to use a closed-form inverse kinematics and a feedback controller with the HUBO $2+$ platform, which was done as a DARPA project in 2013. The solution to this inverse problem involves solving for the joint angles given a desired position and orientation while accounting for singularities, joint limits, and feasible workspace issues [24]. Recently, the reliability and safety research of the submarine robot is also evaluated for control effectiveness about feeding mechanism and the discharge gap detection circuits [25].

Modeling and solving kinematics problems can be laborious, hardware specific, and sometimes computationally expensive. As for traditional imitation control, inverse kinematics problems have to be solved. In this paper, there is no need to solve inverse kinematic problems to find out the joint angles of each of arm and leg. Most robot joint angles are determined from the imitation control except ankle joints. Basically the motion receiving controller needs to do data processing very fast. 
Figure 5 describes the hardware architecture and Figure 6 the software architecture for the motion receiving controller. The receiving motion controller is implemented on ARM processor which is available commercially. For the controller, the software stacks is shown in Figure 6. There are communication protocols TCP/IP stack and USB driver. The developed application program based on Linux has a function to exchange between TCP/IP stack and USB for the serial connection to the robot motion controller.

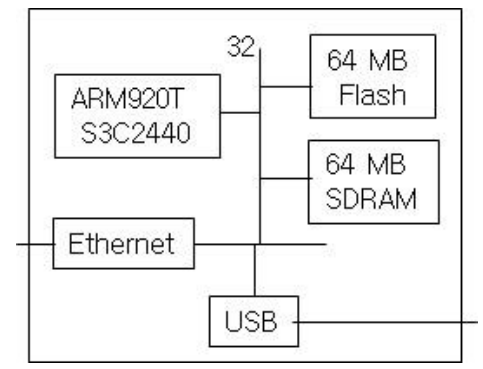

Figure 5. Hardware Architecture

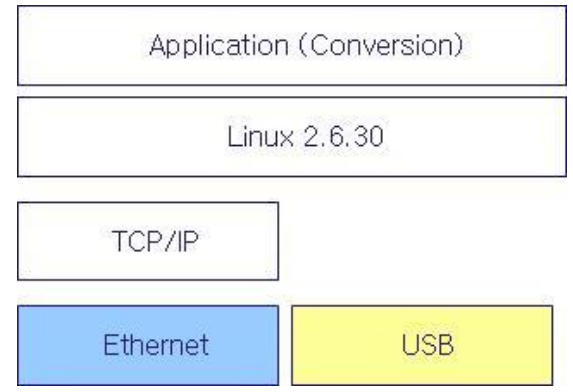

Figure 6. Software Architecture of the Motion Receiving

Thread programming technology [26] is introduced for fast data delivery and saving resources. Technically, a thread is defined as an independent stream of instructions that can be scheduled to run as such by the operating system. To a software developer, the concept of a procedure that runs independently from its main program may be a good concept for a thread. When compared to the cost of creating and managing a process, a thread can be created with much less operating system overhead. Managing threads requires fewer system resources than managing processes. "Joining" with pthread_join() is used to accomplish synchronization between threads. Figure.7 shows the developed motion receiving controller with thread programming.

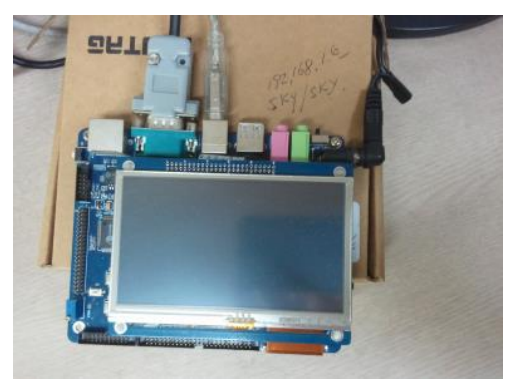

Figure 7. Developed Motion Receiving Controller

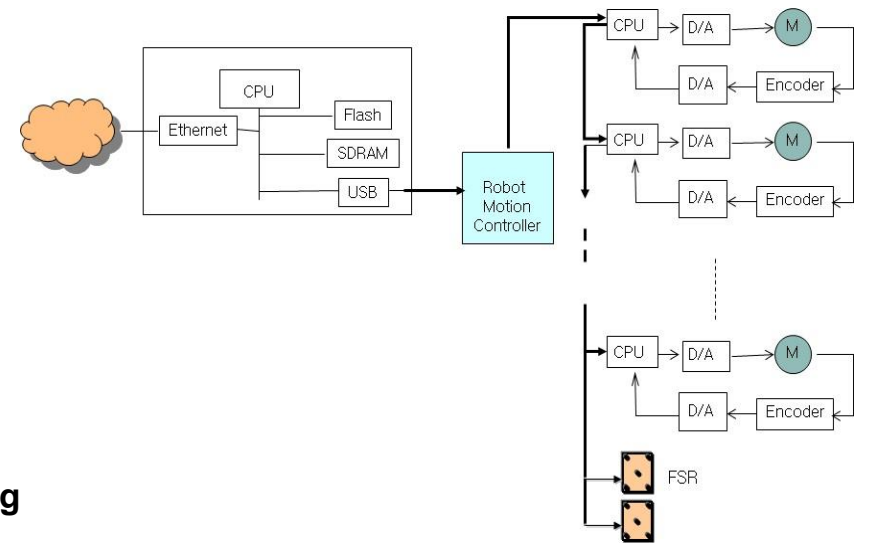

Figure 8. Robot Motion Controller

Figure 8 , describes the connection of the motion receiving controller to the robot motion controller. The robot motion controller can activate several joint ankles, read status values for foot sensors, and communicate with the motion receiving controller.

\section{Experiments and Results}

We have explored how to use the combined controlling scheme of imitation and autonomous with foot sensors and allow an operator to command gaits without 
worrying about kinematics, leaving those tasks to the humanoid robot. For autonomy, foot sensors have been planned to provide walking stability. The key benefits of our approach are that it provides flexibility and intelligence.

We have tested the walking motions with an actual robot on a flat surface. Experiments have been done with the bipedal robot of a total of 17 servomotor joints in its body. It has 17 DOFs (Degree of Freedom) including vertical vibration (pitch), rotation (yaw), and horizontal vibration (roll) DOF. The 17 DOFs of the bipedal robot are 10 for its legs, 6 for its arms, and 1 for its waist. ROBOTIS's Dynamixel AX-12A servomotors are used for the joints. The experimental results demonstrate that the humanoid robot can successfully imitate a series of human walking motions. The operator lets the robot move or stop as he wishes. However, the movement is very slow due to being unstable. A static walking algorithm based on CoP was utilized to perform a few scenarios to learn how well the robot imitates the operator.

In order to show the possibility of controlling a robot in real time, the $\mathrm{CoP}$ and pressure values for the foot sensors are measured and controlling of the ankle joints is done within 0.5 [sec]. Before the robot falls down, it is possible to establish a stable gait through control of the ankle joint servomotors. CoP values on a foot sensor can be traced to control the target robot's gait through the ankle joint of the supporting leg. Figure 9 a) and b) describes how the CoP is traced in case of stable walking and falling down, respectively.

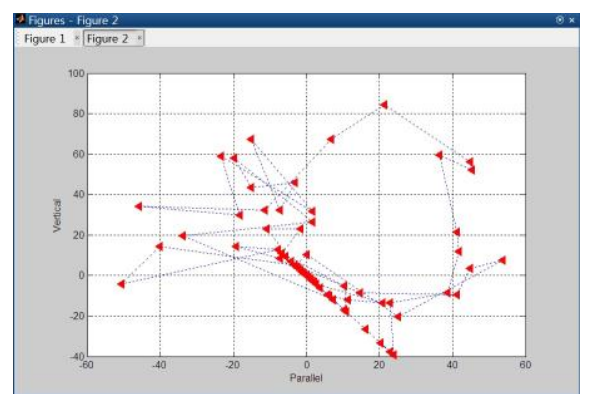

(a) Stable Walking

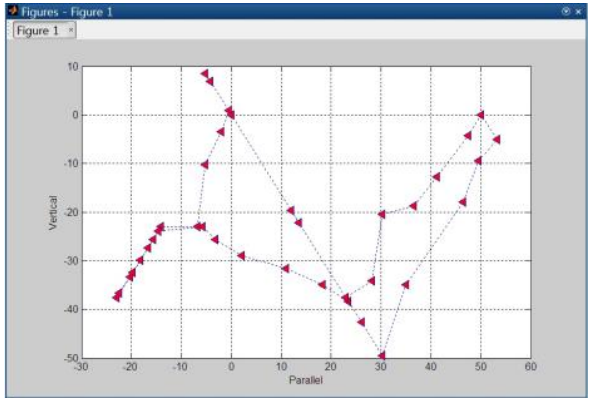

(b) Falling Down

Figure 9. Trace of CoP in Case of a) Stable Walking b) Falling Down

\section{Conclusions}

A human-controlled, humanoid robot that employs imitation control would be intelligent enough to perform difficult jobs because it is controlled by human's intelligence. Because this type of imitation-controlled robot with mobility and intelligence is attractive, there is an increasing amount of research in this area.

Robot walking by using imitation control approach is very useful and convenient because generation of gait and trajectory data for the robot can be produced easily. However, the joint angles generated by the operator's motions are not predictive and cause instability for the postural gait of the robot. For instance, when a foot stands with one leg, the angle of the ankle servomotor is controlled until the robot establishes a stable gait.

With the proposed combination control scheme of imitation and autonomous control, the robot walking is done stably without the use of any a prior analytical or mathematical modeling. Characteristics of the imitation control should be improved by further research. To improve robot walking speed, research for the application of dynamic walking is underway. 
Ideally such a system would allow individual users to quickly and easily tailor their chosen movements and gestures for accurate and intuitive control of any robotic system. Our work can make progress towards applications in hazardous rescue or construction.

\section{Acknowledgments}

This study was conducted by [GRRC (Gyeonggi Regional Research Center) Suwon 2015-B4, Developing technology and systems for coping with situation] as one of the projects of the GRRC, Gyeonggi Province.

This article is a revised and expanded version of a paper entitled Communication System for Internet-based Imitation Control of Humanoid presented at the 2015 International Conference on Future Information \& Communication Engineering held on June 25-26, 2015, in Kota Kinabalu, Malaysia.

\section{References}

[1] R. Goertz and R. Thompson, "Electronically controlled manipulator", Nucleonics, vol. 12, no. 11, (1954), pp 46-47.

[2] http://www.inct-sec.org/actrep/sites/default/files/facilities/LRM.pdf

[3] H. Takanobu, E. Guglielmelli, H. Tabayashi, S. Narita, A. Takanishi, P. Dario, "Remote Interaction between Human and Humanoid Robot", IARP First International Workshop on Humanoid and Human Friendly Robotics, (1998).

[4] K. Hirai, M. Hirose, Y. Haikawa, T. Takenaka, "The development of Honda humanoid robot", Robotics and Automation, 1998 Proceedings. vol. 2, pp 1321 - 1326, 1998.

[5] N. Ee Sian, K. Yokoi, S. Kajita, F. Kanehiro, K. Tanie, "Whole Body Teleoperation of a Humanoid Robot - Development of a Simple Master Device using Joystics, Proceedings of the 2002 IEEE/RSJ, Oct., (2002).

[6] M. Stilman, K.Nishiwaki and S. Kagami, "Humanoid Teleoperation for Whole Body Manipulation", Proceeding of the IEEE Int. Conf. on Robotics and Automation, May, (2008).

[7] P., Gustavo; O., Fernando S. etc. "Genetic Algorithm Applied to Robotic Squad Coordination”, IEEE Press /IEEE Computer Society, V.1, (2009), pp 1-6.

[8] Keio University, Walky project, Graduate School of Media design, Nov. (2009).

[9] A. Senior and S. Tosunoglu, "Design of a Biped Robot", Florida Conference on Recent Advances in Robotics, FCRAR (2006).

[10] D. Le Nguyen and M-E Lee, "OSEK/VDX Porting to the Teo-Wheel Mobile Robot Based on the Differential Drive Method", Journal of Information and Communication Convergence Engineering, vol. 10, no. 4 , Dec. (2012), pp 372- 377.

[11] T. Liu, H. Utsuomiya, Y. Inoue, and K. Shibata, " Synchronous Imitation Control for Biped Robot Based on Wearable Human Motion Analysis System", 2008 IEEE/RSJ International Conference on Intelligent Robots and Systems, France, Sep. 22-26, (2008).

[12] T. Liu, Y. Inoue, K. Shibata, and H. Morioka, "Development of Wearable Sensor Combinations for Human Lower Extremity Motion Analysis", 2006 IEEE International Conference on Robots and Automation, Florida, May. (2006).

[13] A. Ude, C. Man, M. Riley, and G. Christopher. Atkeson. "Automatic generation of kinematic models for the conversion of human motion capture data into humanoid robot motion", In Proc. First IEEE-RAS Int. Conf. Humanoid Robots, (2000).

[14] T. Liu, Y. Inoue, and K. Shibata, "Imitation Control for Biped Robot Using Wearable Motion Sensor", Journal of Mechanisms and Robotics, May, vol. 2, (2010).

[15] S. Ha, Y Bai, and C. Karen Liu, "Human Motion Reconstruction from Force Sensors", ACM SIGGRAPH Symposium on Computer Animation, (2011).

[16] H-Y Liu, W-J Wang, Rong-Jyue Wang, Cheng-Wei Tung, Pei-JuiWang, and I-Ping Chang, “ Image Recognition and Force Measurment Application in the Humanoid Robot Imitation", IEEE Trans. On Instrumentation and Measurement, vol. 61, no. 1, Jan., (2012).

[17] R. Chalodhorn, DB. Grimes, K. Grochow, and R P. N. Rao, "Learning to Walk through Imitation", Twentieth International Joint Conference on Artificial Intelligence, India, Jan. 6-12, (2007).

[18] R. Chalodhorn, et al, "Learning to Walk through Imitation", Twentieth International Joint Conference on Artificial Intelligence, India, Jan. 6-12,( 2007).

[19] N. Miller, O. C. Jenkins, M. Kallman, M. J. Mataric, "Motion capture from inertial sensing for untethered humanoid teleoperation", Proc. Of the 4th IEEE-RAS Intl Conf on Humanoid Robotics, pp $547-565$, (2004). 
[20] H. Song, D. Kim, M. Park and J. Park, "Teleoperation between Human and Robot Arm using Warable Electronic Device", Proceedings of the 17th IFAC World Congress, Seoul, Korea, pp 2430 - 2435, (2008).

[21] J. Aleotti, A. Skoglund and T. Duckett, "Position Teaching of a Robot Arm by Demonstration with a Wearable Input Device", Proc Int; Conf on Intelligent Manipulation and Grasping, (IMG04), (2004), pp 459 - 464.

[22] C. Stanton, A. Bogdanvoych, E. Ratanasena, "Teleoperation of a humanoid robot using full-body motion capture, example movements, and machine learning", Proceedings of Australasian Conference on Robotics and Automation, Dec. (2012).

[23] W-Yo Sim, C-M Chew, Ge-S Hong, "The use of teleoperation for humanoid walking: a first look', Robotics, Automation and Mechatronics, 2004 IEEE Conference on, vol. 1, (2004), pp 1-6.

[24] R O'Flaherty, P. Vieira, M.X. Gret, "Humanoid Robot Teleoperation for Tasks with Power Tools", IEEE Int. Conf. on Tech. for practical Robot Application, TEPRA'13, (2013), pp 1-6 .

[25] Xiaojie Tian, Yonghong Liu, Yunwei Zhang, Rongju Lin and Yuanyuan Xi, "Fault Diagnosis Research of Submarine Casing Cutting Robot for Abandoned Oil Wellhead", International Journal of Security and Its Applications, vol.8, no.1, (2014), pp.213-224,

[26] https://computing.llnl.gov/tutorials/pthreads/

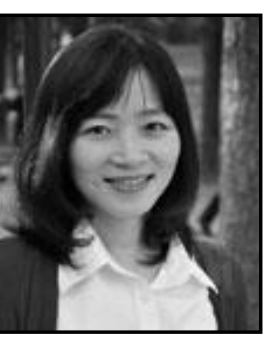

\section{Authors}

Heejung Byun received B.S degree from Soongsil University, Korea, in 1999, M.S. degree from Korea Advanced Institute of Science and Technology (KAIST), Korea in 2001, and the Ph.D. from KAIST in 2005. She was a senior researcher in Samsung Electronics, Ltd. from 2007 to 2010. She is currently a professor with the Department of Information and Telecommunications Engineering, Suwon University, Korea. Her research interests include network protocol, network modeling, controller design, and performance analysis.

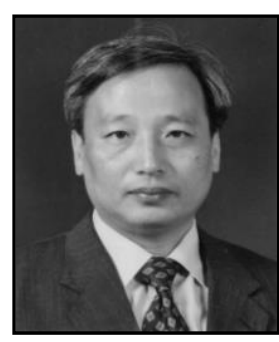

Sugoog Shon received his B.S. (1982) degree in Electrical Engineering from Seoul National University, his M.S. (1984) degree in Electrical Engineering from Seoul National University, and his Ph.D. (1996) in Electrical and Computer Engineering from the University of Texas in Austin. He is a professor in the Department of Information and Telecommunication at the University of Suwon in Korea. His research interests include computer and embedded system, robot, network protocol, network simulation, and network programming. 
International Journal of Security and Its Applications Vol.10, No.1 (2016) 